LIIIAN MARIA LOPES

Rossana Pulcinel Vieira Francisco ${ }^{2}$

MARCELO ZUGAiB ${ }^{3}$

\title{
Anti-inflamatórios e alterações cardíacas fetais
}

\author{
Anti-inflammatory agents and cardiac abnormalities
}

O fechamento ou constrição prematura do canal arterial é uma situação pouco conhecida na prática da obstetrícia, classificada como evento "raro", havendo escassa literatura acerca do assunto que se restringe basicamente a relatos de casos e trabalhos com amostras pequenas ${ }^{1-5}$. Estando seu diagnóstico dependente unicamente da realização de uma ecocardiografia fetal, é compreensível que não exista uma documentação mais extensa sobre o assunto, uma vez que apenas uma pequena porcentagem da população de gestantes em nosso país tem acesso a este exame.

Os anti-inflamatórios não-hormonais $(\mathrm{AINH})$, representados principalmente pelos diclofenacos, agem inibindo a prostaglandina sintetase e são comumente usados na prática clínica para uma variedade de estados dolorosos, principalmente em serviços de pronto atendimento, rotineiramente procurados pelas gestantes em situações de lombalgias, infecção urinária, hemorroidas, etc.

Mais estudada que os diclofenacos, a indometacina, outro AINH usado por muitos anos para inibir trabalho de parto prematuro e para o tratamento do polihidrâmnio, tem comprovado efeito sobre o canal arterial, estando documentada a contrição do canal arterial associada à insuficiência cardíaca grave e aumento de morbidade ${ }^{6,7}$. Um trabalho recente não mostrou efeito colateral significativo consequente ao seu uso, descrevendo apenas $6,5 \%$ de constrição de canal arterial em uma série de 124 gestantes, mas uma "morbidade combinada" de $29 \%$. Esta taxa definitivamente não é baixa, principalmente quando se leva em consideração a combinação de eventos como óbito, sepse, enterocolite necrotizante, hemorragia intraventricular grau 3 ou 4 e insuficiência respiratória ${ }^{8}$.

Cerca de metade dos fetos cujas mães foram tratadas com indometacina por trabalho de parto prematuro, com uma média de idade gestacional de 31 semanas, apresentarão constrição do canal arterial ${ }^{9}$. A proporção de fetos que apresentarão constrição do canal arterial aumenta com a idade gestacional, na qual a medicação é empregada, chegando a $100 \%$ a partir de 34 semanas de gestação ${ }^{10}$. Este estado de constrição é revertido com a interrupção do uso, mas ainda assim pode haver problemas para o feto. Hipertensão pulmonar resultante do aumento de fluxo para os pulmões em razão da constrição ductal se desenvolve em mais da metade dos neonatos expostos à indometacina após a $32^{a}$ semana de gestação ${ }^{11}$, mesmo com uso por curtos períodos (menos de 72 horas) $)^{7,9,11}$. Em um estudo caso-controle, neonatos expostos à indometacina antes da $30^{\mathrm{a}}$ semana de gestação tiveram

Clínica Obstétrica do Hospital das Clínicas da Faculdade de Medicina da Universidade de São Paulo - USP - São Paulo (SP) Brasil.

Médica Assistente Responsável pelo Setor de Cardiologia e Ecocardiografia Fetal da Clínica Obstétrica do Hospital das Clínicas da Faculdade de Medicina da Universidade de São Paulo - USP - São Paulo (SP), Brasil.

2 Professora Doutora do Departamento de Obstetrícia e Ginecologia da Faculdade de Medicina da Universidade de São Paulo - USP - São Paulo (SP), Brasil.

3 Professor Titular de Obstetrícia do Departamento de Obstetrícia e Ginecologia da Faculdade de Medicina da Universidade de São Paulo - USP - São Paulo (SP), Brasil. 
uma incidência maior de hemorragia intracraniana, enterocolite necrotizante, persistência de canal arterial ${ }^{12}$ e diminuição da função renal ${ }^{13}$.

Em 1991, o comitê de análise de efeitos colaterais de drogas (Adverse Reactions Advisory Commitee, ADRAC) enfatizou os riscos de insuficiência cardíaca congestiva e mau prognóstico perinatal relacionados ao uso de AINH na gestação ${ }^{14}$, relatando casos de fechamento ou constrição prematura do canal arterial em gestantes submetidas a tratamento com diclofenaco, indometacina e ácido mefenâmico devido a dor lombar, trabalho de parto prematuro e polidrâmnio, respectivamente. As complicações descritas nestes neonatos foram: cesária de emergência, entubação, acidose metabólica, cianose intensa e até mesmo óbito.

Em 1989, uma gestante com 36 semanas de gestação foi encaminhada para nosso setor de ecocardiografia fetal por cardiomegalia grave ao ultrassom. A ecocardiografia fetal demonstrou dilatação de câmaras direitas, insuficiência tricúspide grave e canal arterial aparentemente fechado. A alteração não permitia que a valva pulmonar se movesse, o que nos levou à hipótese diagnóstica de uma cardiopatia congênita, a "atresia pulmonar". O que era incomum nessa história clínica era o fato de esta gestante ter feito uso de ácido acetilsalicílico em altas doses alguns dias antes em forma de automedicação (2 g ao dia). Após o nascimento, constatou-se atresia pulmonar funcional consequente à hipertensão pulmonar grave por fechamento prematuro do canal arterial, com normalização total dos achados ecocardiográficos após seis meses de vida, constatando-se que a vida fetal é possível mesmo após o fechamento total do canal arterial.

Somente após cinco anos, um segundo caso de constrição importante de canal arterial foi encaminhado a este serviço. Também foi rastreado por cardiomegalia diagnosticada pela ultrassonografia em grávida de 34 semanas que havia recebido diclofenaco injetável por uma semana, prescrito pelo seu obstetra, para tratamento de hemorroida. Seguiram-se outros casos consequentes ao uso tópico de nimesulide gel para tratamento de torcicolo, ao uso de fluoxetina usada para depressão, etc.

Em 22 anos de existência do Setor de Cardiologia e Ecocardiografia fetal da Clínica Obstétrica do Hospital das Clínicas da FMUSP, foi diagnosticado um número significativo de casos de constrição de canal (30 casos, dos quais $67 \%$ estiveram relacionados ao uso de anti-inflamatórios) e que pode ser considerado elevado quando comparado aos dados da literatura, mas que na realidade é modesto se considerarmos que a automedicação e a prescrição médica de anti-inflamatórios é prática rotineira em nosso país, principalmente em serviços de pronto atendimento.

Seriam, então, estes 30 casos ao longo de 20 anos uma expressão da raridade da constrição do canal arterial fetal? Obviamente não. O diagnóstico é subestimado porque o médico que prescreve o anti-inflamatório o faz, na maioria das vezes, sem o conhecimento do risco potencial para a circulação fetal. Nesta situação, o controle seriado do fluxo do canal arterial por meio da ecocardiografia fetal também não costuma ser realizado, o que impede a documentação das consequências deletérias do uso desse medicamento. Imaginamos que muitos médicos têm conhecimento do perigo dos anti-inflamatórios na gestação, mas, ao final, quando se deparam com uma gestante com quadro de dor intensa, pensam resolver o problema da paciente prescrevendo $\mathrm{AINH}$, considerando a possibilidade de ocorrência de constrição de canal arterial um risco apenas teórico. Portanto, se a possibilidade da ocorrência da constrição do canal arterial não é valorizada, menos ainda seria a avaliação dos efeitos adversos com a solicitação da ecocardiografia fetal para seguimento desses casos.

A razão para indicação da ecocardiografia fetal nesses 30 casos de constrição de canal arterial documentados em nosso serviço ao longo destes anos foi, basicamente, cardiomegalia e/ou insuficiência cardíaca em exame ultrassonográfico de rotina ou outras causas não-relacionadas como diabetes, restrição de crescimento fetal ou translucência nucal aumentada. Portanto, o diagnóstico da constrição do canal arterial é acidental e claramente subestimado. Esta situação mostra o desconhecimento médico sobre o assunto e, mais claramente, o abuso da automedicação, tão comum em países em desenvolvimento e sem a informação necessária às gestantes.

Muitos obstetras, amparados apenas pela informação presente na bula da maioria dos AINH, sentem-se confortáveis para usar esta medicação no primeiro e no segundo trimestre, pois seguem à risca que "somente seja empregado na gestação quando houver indicação formal e na menor posologia eficaz. Como outros inibidores da prostaglandinas sintetase, esta orientação aplica-se particularmente nos últimos meses de gestação pela possibilidade de ocorrer inércia uterina e/ou fechamento prematuro do canal arterial". Ao basear-se em conselho tão vago e desconhecendo dados da literatura que, embora escassos, existem, não sabemos ao certo quantos óbitos fetais já foram provocados pelo uso indevido desta medicação durante a gestação.

Sabe-se que nem sempre é possível estabelecer o fator causal nos casos de constrição de canal arterial, sendo então considerados "fechamento espontâneo" 15 -17. Para comprovar a dificuldade em estabelecer associações entre fenômenos, um estudo recente mostrou que os flavonoides, presentes no suco de uva, laranja, maçã, chocolate, chás e chimarrão, elevam de maneira significativa a velocidade do fluxo no canal arterial ${ }^{18}$. Este resultado foi obtido pela comparação 
das velocidades do fluxo no canal arterial de um grupo controle com dieta pobre em flavonoides, com um grupo que ingeria a substância em quantidades superiores ao percentil 75 em relação às quantidades diárias da população local (grupo exposto). Entretanto, como os valores da velocidade do fluxo em canal arterial no grupo exposto, embora maiores, mostraram-se dentro dos limites da normalidade, fica difícil chegar a conclusões com uma casuística tão pequena. À semelhança de estudos nos quais se empregou ácido fólico para a prevenção dos defeitos do tubo neural, a casuística necessária para conclusões relacionadas ao uso de flavonoides e aplicáveis à prática clínica seria de milhares de gestações por se tratar de um evento de prevalência aparentemente baixa ${ }^{19}$.

Portanto, antes de se preocupar com os efeitos dos flavonoides no canal arterial, aparentemente sem repercussão clínica, devemos nos encarregar de divulgar conhecimento e alertar médicos obstetras, socorristas, ortopedistas, urologistas e proctologistas em relação ao perigo da prescrição de anti-inflamatórios para gestantes.

\section{Referências}

1. Zenker M, Klinge J, Kruger K, Singer H, Scharf J. Severe pulmonary hypertension in a neonate caused by premature closure of the ductus arteriosus following maternal treatment with diclofenac: a case report. J Perinat Med. 1998;26(3):231-4.

2. Lopes LM, Zugaib, M. Ductus arteriosus and foramen ovale restriction - diagnosis and etiologic aspects. Cardiol Young. 2001;11 Suppl $1: 225$.

3. Auer M, Brezinka C, Eller P, Luze K, Schweigmann U, Schwarzler P. Prenatal diagnosis of intrauterine premature closure of the ductus arteriosus following maternal diclofenac application. Ultrasound Obstet Gynecol. 2004;23(5):513-6.

4. Luchese S, Mânica JL, Zielinsky P. Estudo da constrição intra-uterina do canal arterial: análise de uma coorte histórica de 20 casos. Arq Bras Cardiol. 2003;81(4):399-410.

5. Paladini D, Marasini $M$, Volpe P. Severe ductal constriction in the third-trimester fetus following maternal self-medication with nimesulide. Ultrasound Obstet Gynecol. 2005;25(4):357-61.

6. Niebyl J, Witter FR. Neonatal outcome after indomethacin treatment for preterm labor. Am J Obstet Gynecol. 1986;155(4):747-9.

7. Moise KJ Jr, Huhta JC, Sharif DS, Ou CN, Kirshon B, Wasserstrum N, et al. Indomethacin in the treatment of premature labor. Effects on the fetal ductus arteriosus. N Engl J Med. 1988;319(6):327-31.

8. Savage AH, Anderson BL, Simhan HN. The safety of prolonged indomethacin therapy. Am J Perinatol. 2007;24(4):207-13.

9. Vermillion ST, Scardo JA, Lashus AG, Wiles HB. The effect of indomethacin tocolysis on fetal ductus arteriosus constriction with advancing gestational age. Am J Obstet Gynecol. 1997;177(2):256-9.

10. Moise KJ Jr. Effect of advancing gestational age on the frequency of fetal ductal constriction in association with maternal indomethacin use. Am J Obstet Gynecol. 1993;168(5):1350-3.

11. Norton ME. Teratogen update: fetal effects of indomethacin administration during pregnancy. Teratology. 1997;56(4):282-92.

12. Norton ME, Merrill J, Cooper BA, Kuller JA, Clyman RI. Neonatal complications after the administration of indomethacin for preterm labor. N Engl J Med. 1993;329(22): 1602-7.

13. Souter D, Harding J, McCowan L, O’Donnell C, McLeay E, Baxendale H. Antenatal indomethacin - adverse fetal effects confirmed. Aust N Z J Obstet Gynaecol. 1998;38(1):11-6.

14. Adverse Drug Reactions Advisory Committee. Premature closure of the fetal ductus arteriosus after maternal use of non-steroidal anti-inflammatory drugs. Med J Aust. 1998;169(5):2701.

15. Hofstadler G, Tulzer G, Altmann R, Schmitt K, Danford D, Huhta JC. Spontaneous closure of the human fetal ductus arteriosus: a cause of fetal congestive heart failure. Am J Obstet Gynecol. 1996;174(3):879-83.

16. Kondo T, Kitazawa R, Noda-Maeda N, Kitazawa S. Fetal hydrops associated with spontaneous premature closure of ductus arteriosus. Pathol Int. 2006;56(9):554-7.

17. Soslow JH, Friedberg MK, Silverman NH. Idiopathic premature closure of the ductus arteriosus: an indication for early delivery. Echocardiography. 2008;25(6):650-2.

18. Zielinsky P, Piccoli AL Jr, Manica JL, Nicoloso LH, Menezes H, Busato A, et al. Maternal consumption of polyphenol-rich foods in late pregnancy and fetal ductus arteriosus flow dynamics. J Perinatol. 2010;30(1):17-21.

19. Czeizel AE, Dudás I. Prevention of the first occurrence of neural-tube defects by periconceptional vitamin supplementation. $\mathrm{N}$ Engl J Med. $1992 ; 327(26): 1832-5$. 